\title{
Suppression of mitochondrial respiration with auraptene inhibits the progression of renal cell carcinoma: involvement of HIF-1a degradation
}

\author{
Yunseon Jang ${ }^{1}$, Jeongsu Han ${ }^{1}$, Soo Jeong Kim ${ }^{1}$, Jungim Kim ${ }^{1}$, Min Joung Lee ${ }^{1}$, \\ Soyeon Jeong ${ }^{1}$, Min Jeong Ryu ${ }^{1,2}$, Kang-Sik Seo ${ }^{3}$, Song-Yi Choi ${ }^{4}$, Minho Shong ${ }^{5}$, \\ Kyu Lim ${ }^{1,2}$, Jun Young Heo ${ }^{1,6}$, Gi Ryang Kweon ${ }^{1,2}$ \\ ${ }^{1}$ Department of Biochemistry, Chungnam National University School of Medicine, Daejeon, Republic of Korea, 301-747 \\ ${ }^{2}$ Research Institute for Medical Science, Chungnam National University School of Medicine, Daejeon, Republic of Korea, \\ 301-747 \\ ${ }^{3}$ R\&D center, KT\&G Life Sciences \\ ${ }^{4}$ Department of Pathology, Chungnam National University School of Medicine, Daejeon, Republic of Korea, 301-747 \\ ${ }^{5}$ Department of Internal Medicine, Chungnam National University School of Medicine, Daejeon, Republic of Korea, 301-747 \\ ${ }^{6}$ Brain research institute, Chungnam National University School of Medicine, Daejeon, Republic of Korea, 301-747 \\ Correspondence to: \\ Gi Ryang Kweon, e-mail: mitochondria@cnu.ac.kr \\ Jun Young Heo, e-mail: junyoung3@gmail.com \\ Keywords: auraptene, renal cell carcinoma, HIF-1a, mitochondrial respiration, elF2a \\ Received: April 03, $2015 \quad$ Accepted: September 29, $2015 \quad$ Published: October 12, 2015
}

\section{ABSTRACT}

Renal cell carcinoma (RCC) progression resulting from the uncontrolled migration and enhanced angiogenesis is an obstacle to effective therapeutic intervention. Tumor metabolism has distinctive feature called Warburg effect, which enhances the aerobic glycolysis rapidly supplying the energy for migration of tumor. To manipulate this metabolic change characteristic of aggressive tumors, we utilized the citrus extract, auraptene, known as a mitochondrial inhibitor, testing its anticancer effects against the RCC4 cell line. We found that auraptene impaired RCC4 cell motility through reduction of mitochondrial respiration and glycolytic pathway-related genes. It also strongly disrupted VEGF-induced angiogenesis in vitro and in vivo. Hypoxia-inducible factor 1a (HIF-1a), a key regulator of cancer metabolism, migration and angiogenesis that is stably expressed in RCCs by virtue of a genetic mutation in the von HippelLindau (VHL) tumor-suppressor protein, was impeded by auraptene, which blocked HIF-1a translation initiation without causing cytotoxicity. We suggest that blockade HIF-1a and reforming energy metabolism with auraptene is an effective approach for suspension RCC progression.

\section{INTRODUCTION}

Nephrectomy is the first-line strategy for curative treatment in the early stage of renal cell carcinoma (RCC). Yet despite this surgery, $30 \%$ of RCC patients face the risk of metastasis to distant sites, including liver, lymph node, brain, bone and pancreas, resulting in a 5-year decrease in survival [1-3]. The aggressiveness of these cancers is accompanied by a metabolic switch toward aerobic glycolysis, called the Warburg effect, which reduces reactive oxygen species (ROS) levels and increases the supply of
ATP [4-6]. As such, this switch is considered indispensable for the migration of RCC cells. Some types of solid cancer with a highly metastatic phenotype, such as RCC and breast cancer, require a high intracellular antioxidant capacity and enhanced ATP generation to support tumor cell migration [7]. Although, it has been reported that mitochondrial complex I inhibitors such as rollinistatin and bullatacin, decrease mitochondrial respiration and ATP generation, thereby inhibiting metastasis of lung and breast cancer cells [8], their effect on RCC has not been studied. Accordingly, suppression of ATP generation by targeting both glycolysis 
and mitochondrial respiration is seen as essential for blocking cancer progression [9]. However, efforts to reverse glycolytic changes in cancers and inhibit mitochondrial respiration are known to produce cytotoxic effects $[9,10]$. Moreover, no modulators with dual mitochondria- and glycolysis-targeting activity that are effective against RCC have been reported.

Auraptene, a natural compound initially isolated and purified from citrus fruit, is known as a mitochondrial complex I inhibitor [11]. In rodent models, auraptene has shown inhibitory effects on mammary and oral carcinogenesis and metastasis of melanoma through modulation of metabolism-related processes, such as hypoxic signaling [12-14]. Auraptene has also been reported to possess apoptogenic activity toward human acute leukemia cells [15]. Although auraptene is among the most useful drugs for regulating tumor progression through modulation of energy metabolism, its efficacy against RCC has not been tested.

Hereditary forms of RCC, including von HippelLindau (VHL) syndrome, account for $2-4 \%$ of total RCC occurrence [16]. The tumor suppressor VHL is an E3 ubiquitin ligase responsible for regulating the degradation of hypoxia-inducible factor $1 \alpha$ (HIF-1 $\alpha$ ). In patients with congenital VHL, a mutation in the $V H L$ gene that inactivates VHL leads to dysregulation of HIF-1 $\alpha$, setting the stage for the development of RCC $[17,18]$. Loss or mutation of the remaining wild-type allele results in constitutive expression of HIF-1 $\alpha$, which is a key modulator of tumor metabolism. Induction of HIF- $1 \alpha$ by hypoxia - a characteristic feature of the tumor environment-promotes the transcription of target genes that lead to invasiveness, metabolic shift, angiogenesis, and metastatic potential [19-21]. Overexpression of HIF- $1 \alpha$ is correlated with metastasis of hepatocellular carcinoma cells [22]. In the current study, we selected auraptene as a candidate modulator of energy metabolism in RCC4 cells through direct targeting of HIF-1 $\alpha$ and mitochondrial respiration, examining its suppressive effects on cancer progression.

\section{RESULTS}

\section{Auraptene inhibits glycolytic and mitochondrial metabolism, but does not affect cell growth}

Because auraptene is an inhibitor of mitochondrial complex I [11], it may be a candidate for the regulation of energy metabolism in RCC. To assess whether mitochondrial oxidative phosphorylation (OXPHOS) in RCC is affected by auraptene, RCC4 cells were incubated with $100 \mu \mathrm{M}$ auraptene or DMSO (vehicle control) for $24 \mathrm{~h}$ and the effects on basal OCR were determined using an XF-24 analyzer. Auraptene significantly inhibited mitochondrial respiration (Fig. 1A), decreasing the basal OCR area under the curve by about $67 \%$ compared to that in DMSO-treated RCC4 cells (Fig. 1B) but did not change in basal ECAR level (Fig. S1). Despite strongly inhibiting mitochondrial complex I, auraptene had no effect on cell viability, as determined by MTT assay (Fig. 1C). Similarly, auraptene treatment did not affect cell growth in the sulforhodamine B (SRB) assay (Fig. 1D).

As auraptene inhibits mitochondrial respiration, cells would require a supply of glycolytic ATP to maintain growth. We found that the mRNAs encoding glucose transporter 1 (GLUT1), hexokinase 2 (HK2), phosphofructokinase (PFK) and lactate dehydrogenase A (LDHA), all key enzymes in the glycolytic pathway, were expressed in RCC4 cells, but quantitative RT-PCR (qPCR) showed that their expression was reduced 30$60 \%$ in the presence of auraptene $(p<0.05$; Fig. $1 \mathrm{E}-1 \mathrm{H})$. Nevertheless, intracellular ATP content and ADP/ATP ratio were unchanged by auraptene treatment (Fig. S2). Collectively, these results indicate that auraptene significantly reduces mitochondrial respiration in RCC cells and slightly suppresses the transcription of glycolytic pathway-related genes without affecting cell growth.

\section{Auraptene decreases RCC4 cell motility and inhibits tube formation by HUVECs}

A reduction in energy metabolism can affect cell motility and angiogenesis as well as cell proliferation [23]. To test whether auraptene has an inhibitory effect on RCC4 cell motility, we performed wound-healing assays. RCC4 cells were cultured in the presence of different concentrations of auraptene or DMSO; then, cell monolayers were wounded by scoring with a pipet tip and the gap width was measured after $24 \mathrm{~h}$ of treatment. Gap closure was decreased by about $20 \%$ in auraptene treated cells compared with DMSO-treated cells (Fig. 2A, 2B), suggesting that auraptene effectively disrupted tumor cell migration. Tube formation by HUVECs is an indicator of tumor angiogenesis [20, 24]. We assessed the effect of different concentrations of auraptene $(0,50,75$ and $100 \mu \mathrm{M})$ on tube formation by HUVECs. HUVECs cultured on Matrigel under hypoxic conditions formed a capillary tube network. Auraptene inhibited tube formation by Matrigel-cultured HUVECs compared with DMSOtreated cells, decreasing the number of branch points at the lowest concentration tested $(50 \mu \mathrm{M})$ and further decreasing the number of branch points at $75 \mu \mathrm{M}$ (by $~ 7$ ) and $100 \mu \mathrm{M}$ (by $\sim 10$ ) (Fig. 2C, 2D). These results indicate that auraptene significantly reduces motility of RCC4 cells and effectively inhibits tube formation by HUVECs.

\section{Auraptene inhibits VEGF-induced neovascularization in vivo}

To verify that auraptene has anti-angiogenic activity, we performed the Matrigel plug assay in vivo. A mixture of Matrigel, vascular endothelial growth factor (VEGF) and 

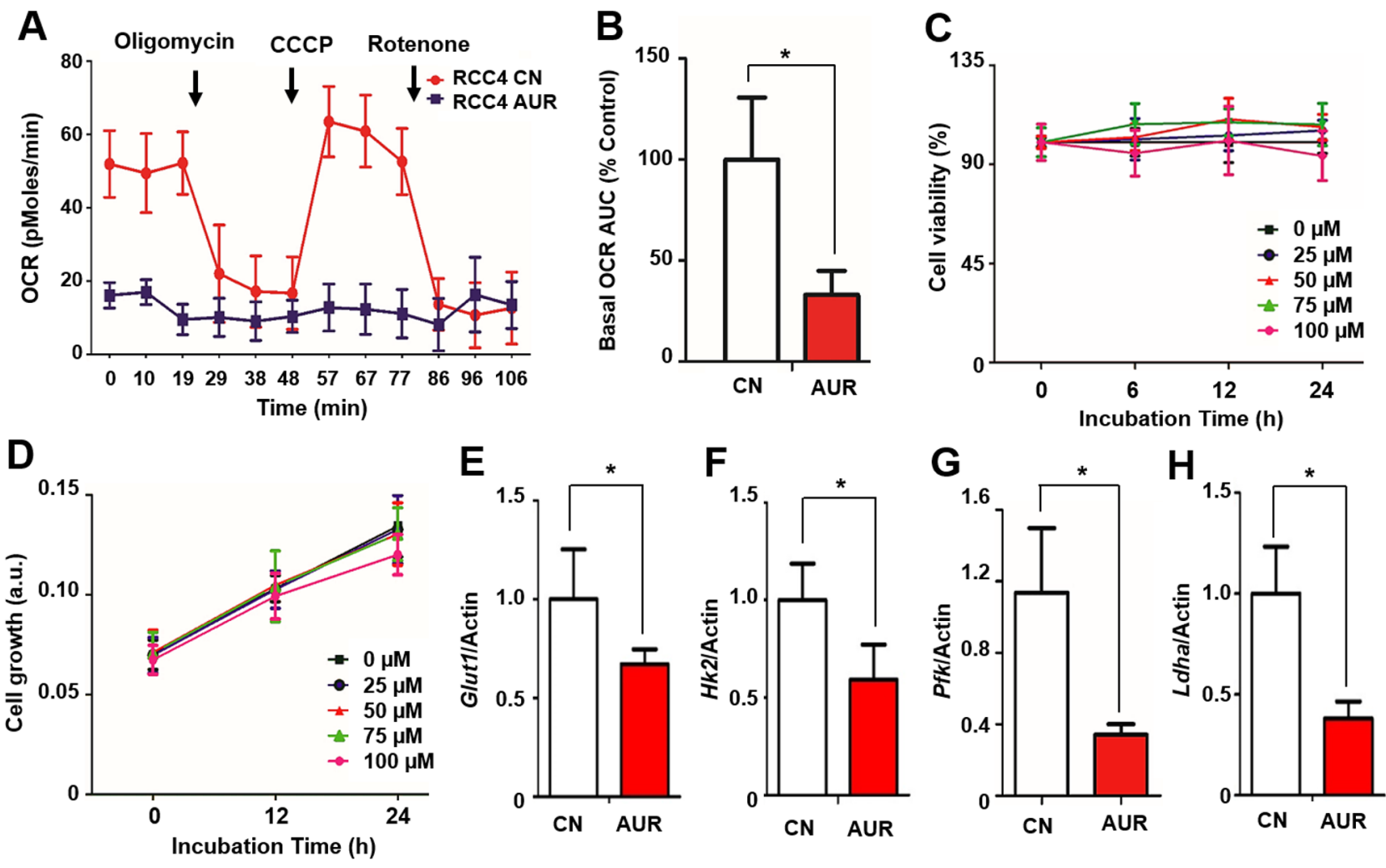

Figure 1: Auraptene significantly reduces the OCR of RCC cells and disrupts transcription of HIF-1 $\alpha$ target genes without affecting cell viability. A. The OCR of RCC4 cells was measured using an XF24 analyzer after incubation with DMSO or $100 \mu \mathrm{M}$ auraptene for $24 \mathrm{~h}$. Arrows indicate times at which mitochondrial inhibitors $(2 \mu \mathrm{g} / \mathrm{ml}$ oligomycin, $5 \mu \mathrm{M} \mathrm{CCCP}$, and $2 \mu \mathrm{M}$ rotenone $)$ were added to media. B. Basal OCR area under the curve was calculated using XF24 software. C, D. Effects of 0, 25, 50, 75, and $100 \mu \mathrm{M}$ auraptene on RCC4 cell viability after the indicated times was determined by MTT assay and SRB, respectively. Viability is presented as a percentage relative to zero time. E-H. mRNA expression of GLUT, HK2, LDHA and PFK in RCC4 cells cultured in the presence or absence of $100 \mu \mathrm{M}$ auraptene for $24 \mathrm{~h}$ was assessed by qPCR. Values are expressed as fold change. $\beta$-Actin was used as control. Data are presented as means $\pm \mathrm{SD}$ (bars) of triplicate samples $(* P<0.05)$.

either DMSO or $100 \mu \mathrm{M}$ auraptene was subcutaneously injected to the flanks of nude mice. Seven days later, the plugs was detached from the skin and visualized. In the absence of auraptene, the plugs had a red color due to the infiltration of vessels (Fig. 3A). Neovascularization was quantified by measuring the hemoglobin content of the plugs, with the hemoglobin content of each plug represented as a percentage of control. Plugs in the auraptene treated group had a 5-fold lower hemoglobin content than control plugs (Fig. 3B), and a lower density of infiltrated blood vessels. VEGF-induced angiogenesis is crucial for tumor progression $[24,25]$ and antiangiogenic agents have been used to inhibit aggressive tumor spread. We verified whether auraptene affects the transcription of $V e g f-a$, finding that auraptene reduced Vegf- $a$ mRNA level by $\sim 70 \%$, as shown by qPCR analysis (Fig. 3C). These results indicate that auraptene effectively inhibited VEGFinduced angiogenesis in vivo.

To determine whether auraptene inhibited angiogenesis in vivo, $1 \times 10^{7}$ RCC4 cells were subcutaneously injected into 5 week old nude mice, followed nine days later by the intratumoral injection of auraptene every other day (Fig. 3D). We found that auraptene treated mice had reduced tumor growth, whereas tumors injected with DMSO showed a gradual increase in size (Fig. 3E). After 25 days, the mice were sacrificed and tumor weight and vascularization status were analyzed. As expected, tumor weight (Fig. 3F) and the number of well-vascularized vessels (Fig. 3G, $3 \mathrm{H})$ were each about one-third lower in auraptene- than in DMSO-treated mice. Immature vessels with reduced diameter, blood leakage represented by red blood cells and stained with brown colored hemosiderin were observed in auraptene injected group compared to the well-formed vessels of DMSO-treated mice with basement membrane and pericytes surrounding vessel wall. (Fig. 3G). Consistent with H\&E staining results, immunofluorescence showed that auraptene reduced the number of CD31 positive cells but increased heterogeneity (Fig. 3I, 3J). Taken together, these findings indicate that auraptene can inhibit vessel formation, suppressing tumor cell growth. 

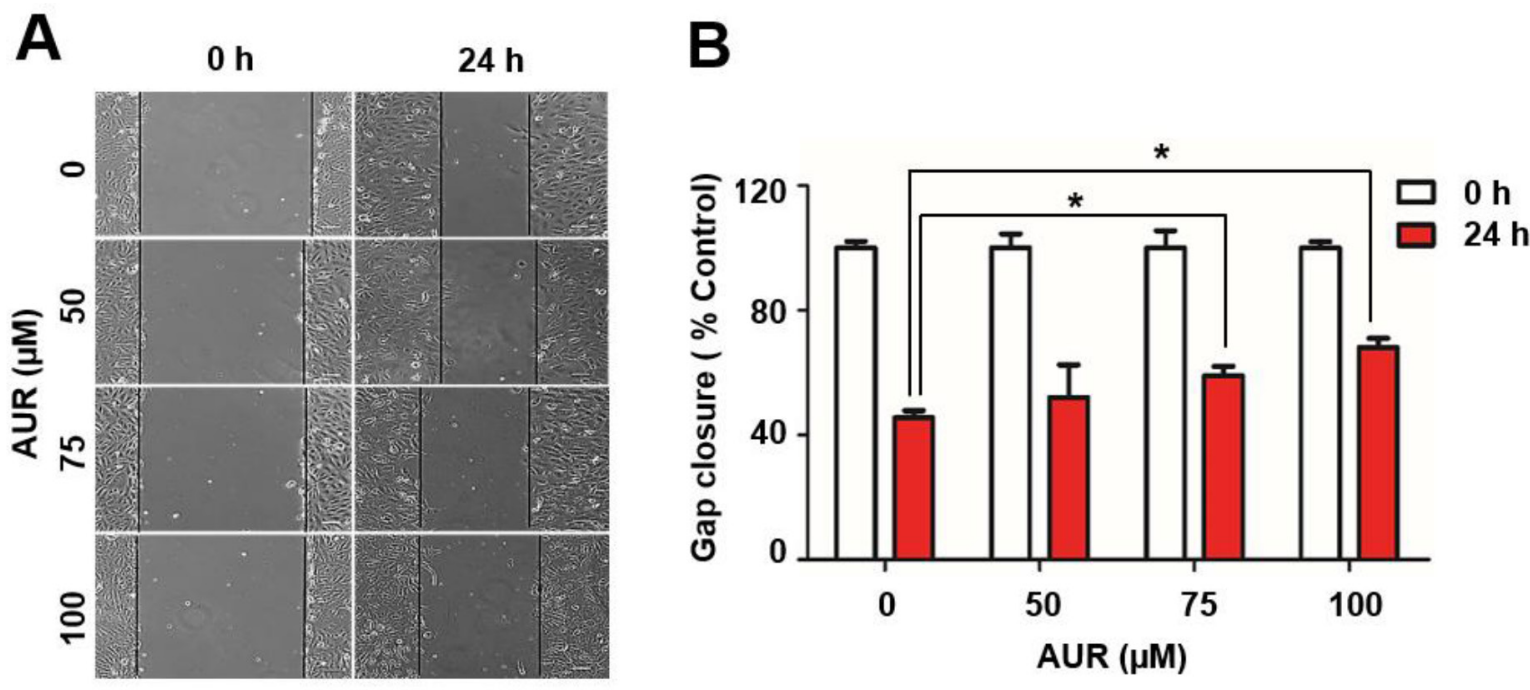

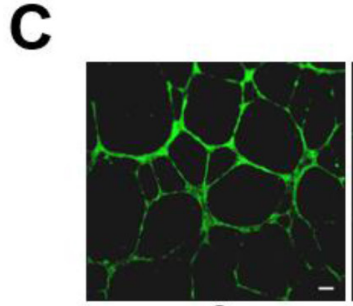

0

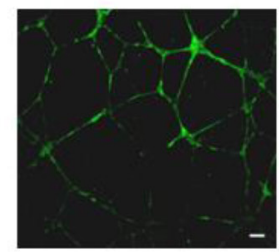

75

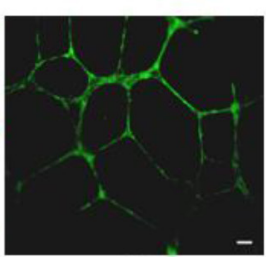

50

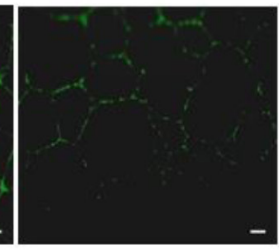

100

AUR $(\mu \mathrm{M})$

D

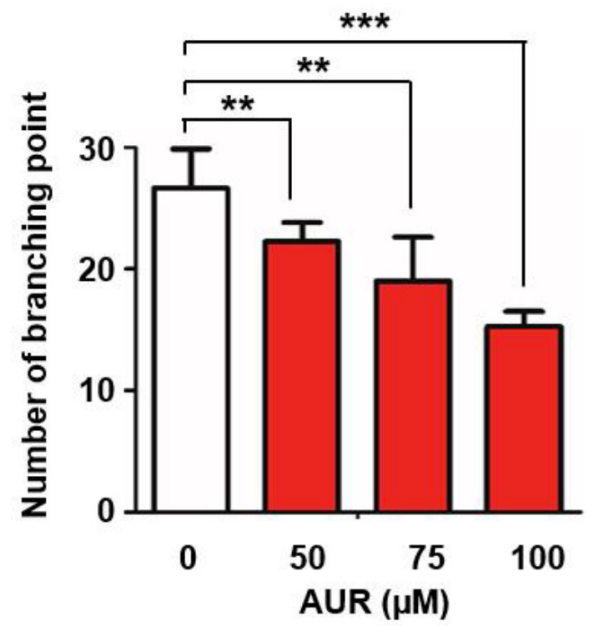

Figure 2: Auraptene delays RCC4 cell migration and inhibits tube formation by HUVECs. A. RCC4 cell wound-healing assays were performed in the presence or absence of $100 \mu \mathrm{M}$ auraptene after $24 \mathrm{~h}$. B. Gap closure was measured and presented as gap distance relative to controls (as a percentage). C. Effects of auraptene on angiogenesis were verified by HUVEC tube-formation assays. HUVECs were plated on Matrigel and incubated under hypoxic $\left(1 \% \mathrm{O}_{2}\right)$ conditions. Tube formation was visualized with the fluorescent dye, calcein-AM, $24 \mathrm{~h}$ after auraptene treatment. Scale bars: $10 \mu \mathrm{m}$. D. The number of branching points was counted. Data are presented as means \pm SD (bars) of triplicate samples $(* P<0.05, * * P<0.01 ; * * * P<0.001)$.

\section{Auraptene induces HIF-1 $\alpha$ degradation in a VHL-independent manner, and HIF-1 $\alpha$ knockdown delays RCC4 cell migration}

HIF-1 $\alpha$ is involved in promoting vessel formation through induction of VEGF expression and enhancing tumor growth through upregulation of glycolytic pathwayrelated genes $[20,26,27]$. To test the role of HIF- $1 \alpha$ in mediating the effects of aurapene, we first assessed HIF-1 $\alpha$ transcription levels by qPCR, but found no significant change in HIF-1 $\alpha$ mRNA level with auraptene treatment (Fig. S3). Next, we assessed whether auraptene affected HIF-1 $\alpha$ protein levels in RCC4 cells, which constitutively express HIF- $1 \alpha$ protein owing to a deficiency of VHL
$[17,28]$. Unlike the case for murine embryonic fibroblasts (MEFs), HepG2 human hepatic carcinoma cells and MCF-7 human breast cancer, HIF-1 $\alpha$ was notably elevated in RCC4 cells, even under normoxic conditions, and was prominently localized in the nucleus (Fig. S4A, S4B). In RCC4 cells treated for $24 \mathrm{~h}$, auraptene decreased HIF- $1 \alpha$ protein levels in a concentration-dependent manner (Fig. 4A). Over this same time frame, treatment with $100 \mu \mathrm{M}$ auraptene resulted in the time-dependent degradation of HIF-1 $\alpha$ (Fig. 4B). To test whether auraptene affected HIF-1 $\alpha$ induction in the context of hypoxic conditions representative of the tumor environment, we reconstituted VHL in RCC4 cells, which lack endogenous VHL, and assessed the effects of auraptene $(100 \mu \mathrm{M})$ 
A

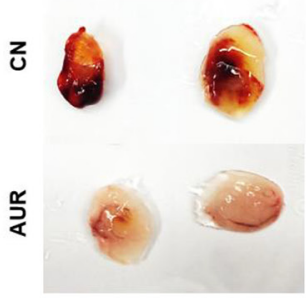

F

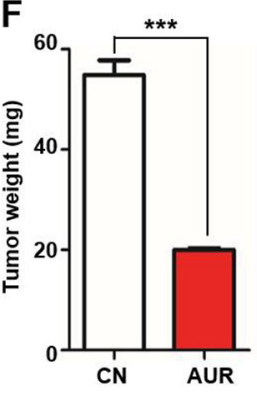

B

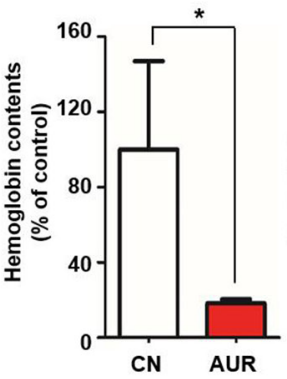

C

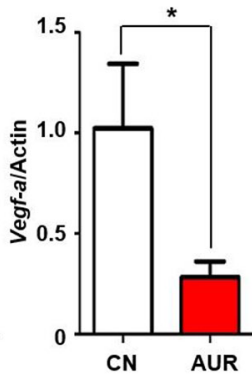

$\mathbf{G}$

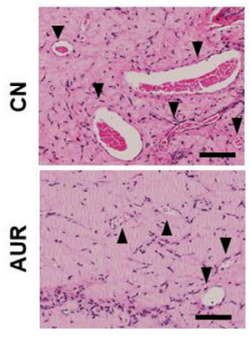

H
D
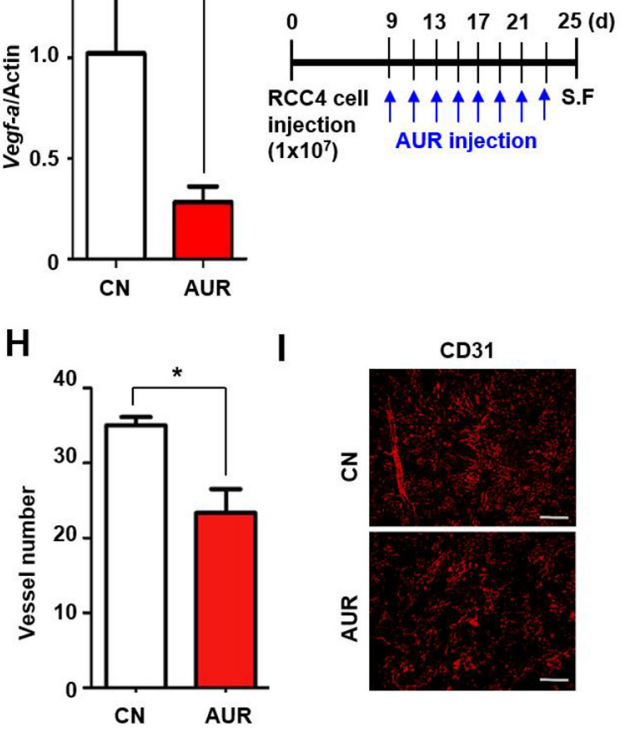

I

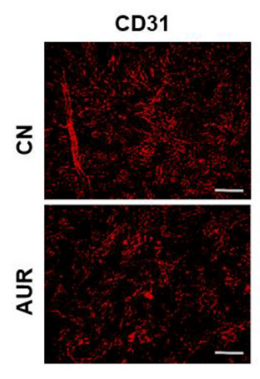

E

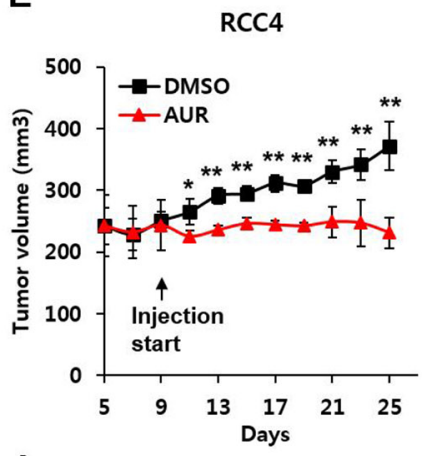

J

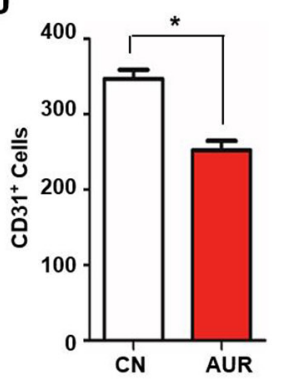

Figure 3: Auraptene inhibits VEGF-induced angiogenesis in vivo. A. VEGF-induced angiogenesis was assessed by the Matrigel plug assay. A Matrigel mixture containing DMSO or $100 \mu \mathrm{M}$ auraptene was subcutaneously injected into 6 week old male immunodeficient mice ( $n=5$ per group). After 7 days, the Matrigel plug was detached from the skin and photographed. B. Blood extracted from Matrigel was added to Drabkin's solution, and the absorbance at OD $450 \mathrm{~nm}$ was measured. The hemoglobin contents of each plug were represented as $\%$ of control. C. $V E G F-A$ mRNA expression in RCC4 cells cultured in the presence or absence of $100 \mu \mathrm{M}$ auraptene for $24 \mathrm{~h}$, as assessed by qPCR analysis. Values were normalized to those of $\beta$-actin. Data are presented as means \pm SDs (bars) of triplicate samples $\left({ }^{*} P<0.05\right.$ ). D. Effect of auraptene on RCC4 xenografts. RCC4 cells (1x10 RCC4) were subcutaneously injected to the flank of 5 week old nude mice. After 9 days, auraptene or DMSO was injected into the tumor every other day ( $n=10$ per group). E. tumor size measured by calipers every other day and calculation of tumor volumes. F. tumor weight. G. H\&E staining of vessels from xenografts after 25 days. Arrows indicate the vessels. H. numbers of vessels. I. immunofluorescence images of CD31 of DMSO- and auraptene-injected tumors. J. Counting of CD31 positive cells. The graph represents the mean \pm SEM (bars) of 5 mice in each group. Scale bar in G is $100 \mu \mathrm{m}$ and $\mathrm{H}$ is $200 \mu \mathrm{m}$.

on these RCC4/VHL cells under normoxic $\left(20 \% \mathrm{O}_{2}\right)$ and hypoxic $\left(1 \% \mathrm{O}_{2}\right)$ conditions. As shown in Fig. $4 \mathrm{C}$, auraptene strongly decreased HIF-1 $\alpha$ protein levels in RCC4/VHL cells under hypoxic conditions, and also inhibited mitochondrial respiration (Fig. S5). These results suggest that auraptene induces HIF-1 $\alpha$ downregulation regardless of the presence or absence of VHL. To determine whether the inhibitory effect of auraptene on cell migration is a consequence of HIF- $1 \alpha$ inhibition, we transfected RCC4 cells with a siRNA targeting a Hif- $1 \alpha$ consensus RNA and performed wound-healing assays. Knockdown of HIF-1 $\alpha$ was confirmed by Western blotting (Fig. S6). Following wounding and treatment with auraptene, gap distance was increased more than 2-folds in siHif-1 $\alpha$-transfected cells compared with cells transfected with a scrambled control siRNA (Fig. 4D, 4E).

\section{Auraptene promotes HIF-1 $\alpha$ protein degradation by inhibition of translation initiation}

To determine whether the decrease in HIF- $1 \alpha$ levels was attributable an increase in HIF-1 $\alpha$ protein degradation (decreased half-life) or a decrease in translation, we first evaluated proteasome-mediated degradation of HIF-1 $\alpha$. In RCC4 cells treated with DMSO (vehicle control), co-treatment the proteasome inhibitor MG132 resulted in the accumulation of HIF-1 $\alpha$. Notably, however, even with the proteasome inhibited with MG132, co-treatment with auraptene still significantly decreased HIF-1 $\alpha$ levels compared with DMSO co-treatment (Fig. 5A). Interactions with heat shock protein 90 (HSP90) are important for HIF-1 $\alpha$ protein stability, with dissociation from HSP90 leading to proteasome-mediated degradation of HIF-1 $\alpha$ [29, 30]. To determine whether auraptene acted by interfering with the association of HIF-1 $\alpha$ with HSP90, we overexpressed HSP90 in RCC4 cells by transfecting cells with a FLAG-HSP90 expression construct and tested the interaction of HIF-1 $\alpha$ with HSP90 in the presence and absence of auraptene. We found that auraptene had no effect on the association of HIF-1 $\alpha$ with HSP90 (Fig. S7). Taken together, these data suggest that auraptene-induced HIF- $1 \alpha$ down-regulation is not dependent on proteasome-mediated degradation. We did find, however, that auraptene decreased the halflife of HIF-1 $\alpha$ protein in RCC cells, showing that, in the presence of cycloheximide to inhibit de novo protein 


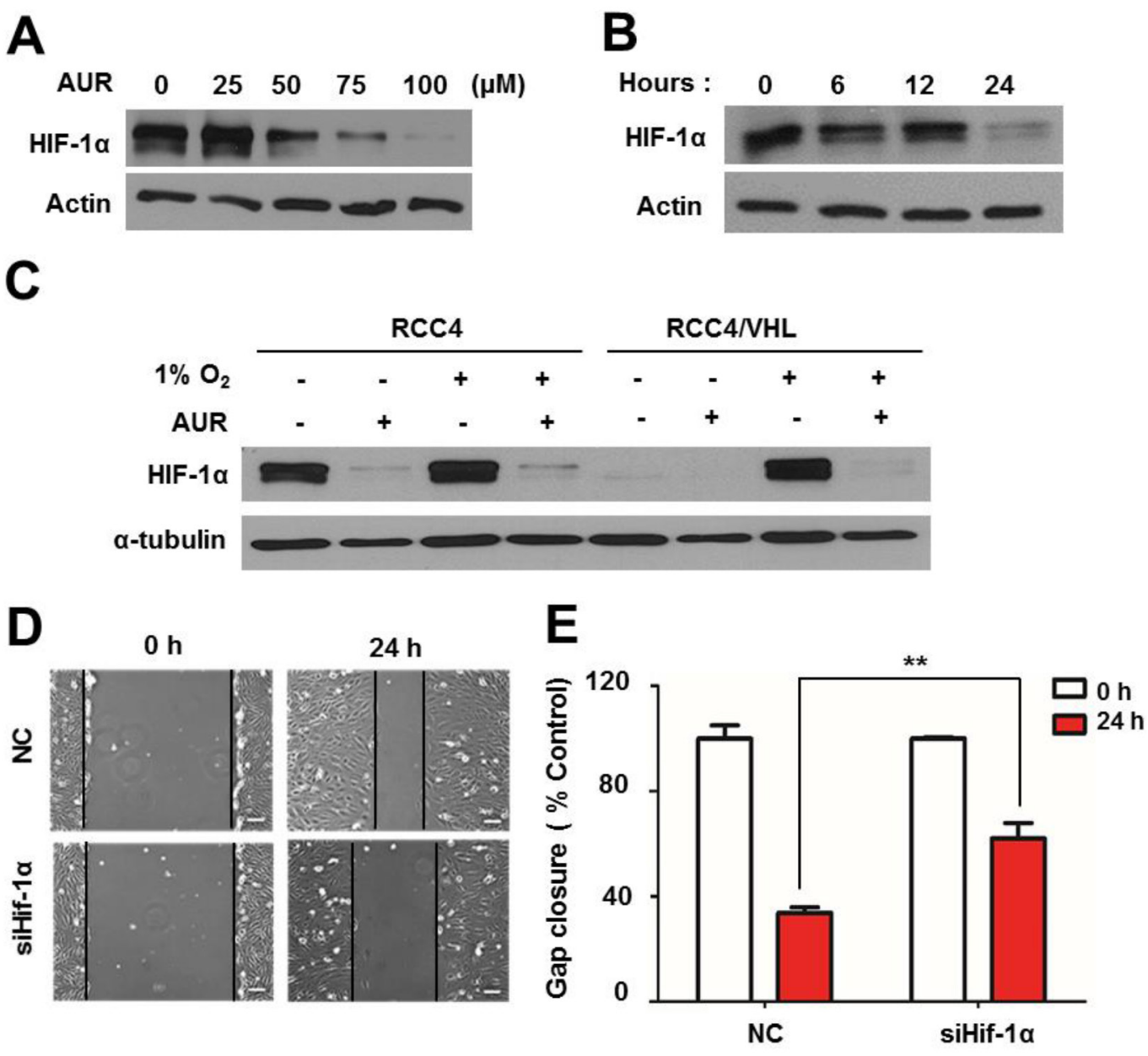

Figure 4: Auraptene induces HIF-1 $\alpha$ degradation in a VHL-independent manner, and siRNA-mediated HIF-1 $\alpha$ knockdown delays RCC4 cell migration. A. RCC4 cells were incubated for $24 \mathrm{~h}$ in the absence or presence of $0,25,50,75$ or 100 $\mu \mathrm{M}$ auraptene (AUR). B. RCC4 cells were cultured in the absence or presence of AUR $(100 \mu \mathrm{M})$ for $0,6,12 \mathrm{or} 24 \mathrm{~h}$. C. RCC4 cells and RCC4/VHL cells were incubated for $24 \mathrm{~h}$ in the presence or absence of $100 \mu \mathrm{M}$ auraptene under normoxic or hypoxic conditions. Protein levels of HIF-1 $\alpha$ and $\alpha$-tubulin were assessed by Western blotting. D. Effects of siHif-1 $\alpha$ on cell migration were verified. E. Average distance to the opposing side of the wound was measured $24 \mathrm{~h}$ after siHif- $1 \alpha$ transfection and expressed as gap distance relative to controls (as a percentage). Data are presented as means $\pm \mathrm{SD}$ (bars) of triplicate samples $(* * P<0.01)$. NC, negative control.

synthesis, HIF-1 $\alpha$ protein levels decreased more rapidly in the presence of auraptene (Fig. 5B, 5C). Finally, we examined whether the effects of auraptene on HIF-1 $\alpha$ levels involved eukaryotic translation initiation factor $2 \alpha$ (eIF $2 \alpha$ ), a component of the eIF2 ternary complex that is known to initiate HIF-1 $\alpha$ gene translation [31]. In RCC4 cells and HeLa cells grown under hypoxic conditions, treatment with auraptene significantly increased the levels of phosphorylated eIF2 $\alpha$ (p-eIF2 $\alpha$ ) (Fig. 5D and Fig. S8). These results suggest that auraptene down-regulates HIF- $1 \alpha$ protein by inhibiting its translation initiation rather than by promoting its degradation (Fig. 5E). Notably, auraptene did not affect cleavage of poly-(ADPribose) polymerase (PARP), an apoptosis marker protein, in HeLa cells (Fig. S8), further supporting the conclusion that auraptene is not cytotoxic.

\section{DISCUSSION}

About $30 \%$ of patients with RCC, a potentially fatal type of solid tumor, suffer from uncontrolled metastasis and chemoresistance [1-3]. RCC metastasis is starting from enhanced migration and it is not restricted to lymph nodes around the kidney, but can spread to distant organs, such as the pancreas, bone, and brain. To date, interferon (IFN), sunitinib and bevacizumab, and interleukin (IL)-2 have been the main options for treating RCC and preventing recurrence. Unfortunately, response rates for these drugs in preventing metastasis and recurrence are only $\sim 15 \%$ [32]. Given this limited efficacy, clinicians have turned to other therapeutics, including sorafenib and pazopanib-inhibitors of vascular endothelial growth factor A (VEGF-A) that target tumor 
B
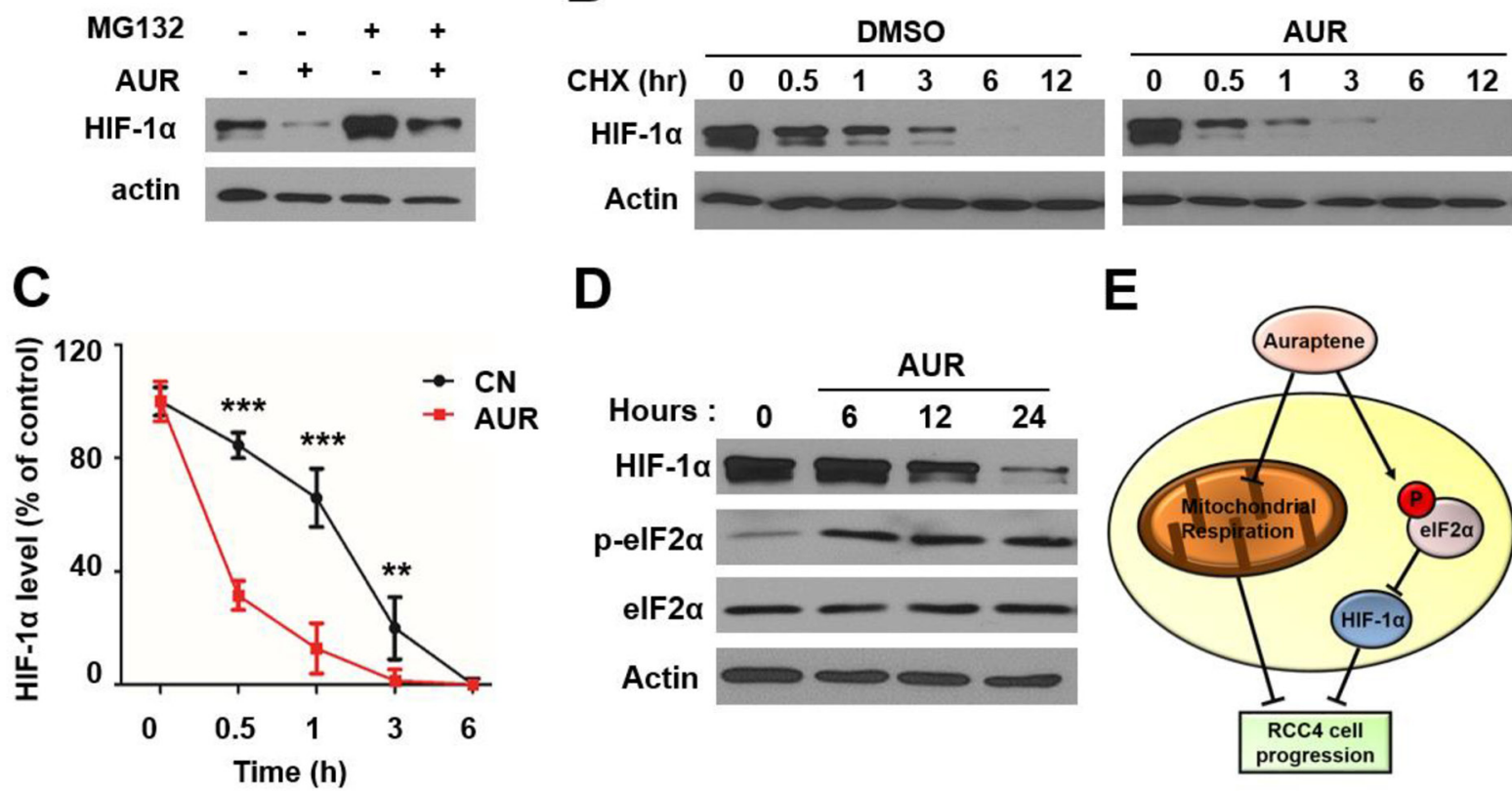

D
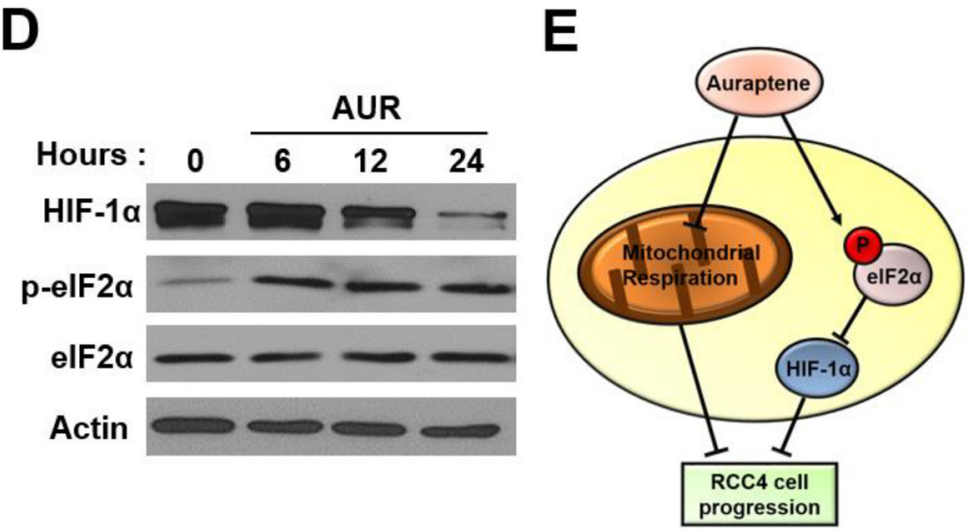

Figure 5: Auraptene significantly decreases HIF-1 $\alpha$ protein through translational inhibition. A. RCC4 cells were incubated in the absence or presence of $100 \mu \mathrm{M}$ auraptene and $20 \mu \mathrm{M}$ MG132 for $24 \mathrm{~h}$. B. RCC4 cells were cultured in the absence or presence of $100 \mu \mathrm{M}$ auraptene and $40 \mu \mathrm{g} / \mathrm{mL}$ CHX, added to media at the indicated incubation times. HIF- $1 \alpha$ and $\beta$-actin levels were analyzed by Western blotting. C. Band intensity was measured using the ImageJ program. Data are presented as means \pm SD (bars) of triplicate samples $(* * P<0.01 ; * * * P<0.001)$. D. Protein levels of HIF- $1 \alpha$, total eIF $2 \alpha$, and $p$-eIF2 $\alpha$ were determined by Western blotting after administration of auraptene. $\beta$-Actin was used as a loading control. E. Schematic depiction of inhibition of angiogenesis and motility of RCC cells by auraptene through regulation of HIF- $1 \alpha$ and eIF $2 \alpha$.

angiogenesis - and multi-tyrosine kinase inhibitors that target platelet-derived growth factor (PDGFR)- $\alpha$ or VEGFR-1. However, these drugs are unable to overcome chemoresistance and leukopenia owing to their cytotoxic effects [33-35]. In the present study, we investigated the anticancer properties of auraptene, isolated from citrus fruit, against RCC. We found that auraptene significantly suppressed tumor energy metabolism, leading to inhibition of motility and it did so without causing cytotoxicity.

Cancer cells favor anaerobic glycolysis as the metabolic pathway for obtaining ATP, although mitochondrial OXPHOS still provides low amounts of ATP [9]. This preference has motivated trials to reverse the aberrant metabolism in cancer cells using drugs such as the $\alpha$-tocopherol derivative, ESeroS-GS [9, 36]. However, it has been reported that $\alpha$-tocopherol derivatives and combined administration of glycolysis and mitochondrial inhibitors are associated with cytotoxicity [8, 37]. In contrast, one previous study reported that administration of auraptene was cytotoxic toward breast cancer cells only under serum-starved conditions. Furthermore, against the Jurkat $\mathrm{T}$ cell line, auraptene showed cytotoxic effects only at high concentrations $(>150 \mu \mathrm{M})[15]$. According to the previous report, auraptene content of dried peel powder of Citrus Kawachiensis was defined to $4.07 \pm 0.033 \mathrm{mg} / \mathrm{g}$ [38]. They daily administered 32 and $64 \mathrm{mg} / \mathrm{kg}$ of auraptene to mouse orally and it showed beneficial effect for defending inflammation. We have mostly used $100 \mu \mathrm{M}$ auraptene in current study, which is obtainable using the concentrated powder and it did not show toxicity at this concentration. Notably, although auraptene treatment suppressed mitochondrial respiration and glycolytic pathway-related genes in RCC cells, it did not affect cell viability at concentrations less than $100 \mu \mathrm{M}$. In line with the cell viability result, ATP content and ADP/ATP ratio did not changed and we can predict the alternative pathway of glycolysis compensate energy depletion such as TCA cycle and beta oxidation (Fig. S9). Moreover we cannot find any Tunnel positive cells by treatment of auraptene in vivo (Fig. S10A, S10B). Accordingly, auraptene could be a candidate for inhibiting cancer progression with no associated cytotoxicity.

The enhancement of VEGF-induced angiogenesis is observed in stages of tumor progression and therapeutic strategies targeting neovascularization of tumor has been tried [25]. So, we assessed whether auraptene had an antiangiogenic effect. We found that auraptene directly decrease VEGF mRNA expression. Importantly, 
auraptene effectively inhibited tube formation by HUVEC cells under hypoxic condition. Also, auraptene inhibited VEGF-induced neovascularization by 10 -fold in vivo. Tumor cell motility and neovascularization are necessary for RCC progression, a process that requires rapid availability of an abundant energy supply. According to the Warburg effect, most of the energy used is supplied by glycolysis. Consistent with this hypothesis, HK2 and GLUT1 are also highly expressed in many tumors, including RCCs, and mediate enzymatic and transport processes involved in glycolysis [39]. Furthermore, LDHA contributes to aerobic glycolysis in tumors. The abovementioned glycolysis-related genes are targeted by the transcription factor, HIF-1 $\alpha$. Unlike other tumors, which express HIF-1 $\alpha$ only under hypoxic conditions, RCCs express high levels of HIF-1 $\alpha$ under normoxic conditions. We found that auraptene abolished HIF-1 $\alpha$ protein translation and reduced transcription of HIF-1 $\alpha$ target genes, including GLUT2, HK2 and $L D H A$, which are involved in regulating tumor metabolism [5, 19, 21]. This ablation of HIF-1 $\alpha$ by auraptene was further associated with inhibition of angiogenesis, as reflected in reduced endothelial tube formation and decreased expression of $V E G F$, as well as suppression of RCC cell migration. As expected, siRNA-mediated HIF-1 $\alpha$ knockdown resulted in inhibition of RCC cell motility. Consistent with these findings, small molecule inhibitors of HIF-1 $\alpha$ such as PX-478 and geldanamycin have been shown to reduce tumor cell proliferation and angiogenesis [29, 40, 41]. However, unlike auraptene, these compounds also induce apoptosis.

EIF $2 \alpha$ is known to regulate HIF-1 $\alpha$ gene translation [31]. Phosphorylation on Ser51 of the eIF2 $\alpha$ subunit blocks formation of the eIF2 ternary complex, resulting in inhibition of HIF-1 $\alpha$ translation [40]. Aminoflavone, a ligand of the aryl hydrocarbon receptor, is known to repress HIF- $1 \alpha$ translation in human breast cancer cells by inducing eIF2 $\alpha$ phosphorylation [42]. Hydrogen sulfide $\left(\mathrm{H}_{2} \mathrm{~S}\right)$ is also reported to regulate HIF$1 \alpha$ through eIF $2 \alpha$ phosphorylation. This study showed that auraptene significantly induced phosphorylation of eIF $2 \alpha$ beginning at $6 \mathrm{~h}$, and that acute treatment with auraptene rapidly (within $7 \mathrm{~min}$ ) inhibited mitochondrial respiration (Fig. S11A, S11B) and simultaneously increased ECAR (Fig. S11C, S11D). Although we did not specifically address the temporal relationship between mitochondrial inhibitory function and eIF $2 \alpha$ phosphorylation, we expect that suppression of mitochondrial energy metabolism proceeds to HIF-1 $\alpha$ degradation within $6 \mathrm{~h}$. Inhibitors of HIF-1 $\alpha$ transactivation carry a risk of causing coagulative necrosis and cytotoxicity [43]. However, because HIF-1 $\alpha$ is not the initial target, auraptene can more effectively inhibit cellular motility and tube formation without causing cytotoxicity. Auraptene effect on HIF-1 $\alpha$ downregulation was also verified using $\mathrm{HeLa}$ cells grown under hypoxic $\left(\begin{array}{lll}1 \% & \mathrm{O}_{2}\end{array}\right)$ conditions. Additionally, auraptene significantly induce phosphorylation of eIF $2 \alpha$ in a dose dependent manner (Fig. S8). These finding suggests that anticancer property of auraptene through inhibition of HIF- $1 \alpha$ is not restricted to renal cell carcinoma. Taken together, our results demonstrate that auraptene exerts a suppressive effect on the progression of RCC4 cells by strongly inhibiting mitochondrial respiration and directly targeting HIF-1 $\alpha$ signaling, without producing cytotoxic effects.

\section{MATERIALS AND METHODS}

\section{Cell culture}

RCC4 and RCC4/VHL human RCC cells were purchased from ECACC (Salisbury, UK). As instructors indicated, RCC4 cells were stably transfected with pcDNA3 vector and RCC4/VHL cells were transfected with pcDNA3-VHL, conferring neomycin resistance. Cells were maintained in Dulbecco's Modified Eagle Medium (DMEM) containing 10\% fetal bovine serum (FBS), $1 \%$ penicillin/streptomycin, and $100 \mu \mathrm{g} / \mathrm{ml} \mathrm{G418}$ at $37^{\circ} \mathrm{C}$ in a humidified $5 \% \mathrm{CO}_{2} / 21 \% \mathrm{O}_{2}$ environment. Low-oxygen conditions were created by adjusting the environment to $1 \%$ oxygen, $5 \% \mathrm{CO}_{2}$, and $94 \% \mathrm{~N}_{2}$ using a hypoxia chamber (New Brunswick Scientific, CT, USA). Auraptene was dissolved in dimethyl sulfoxide (DMSO). Cycloheximide was purchased from SigmaAldrich, and MG132 was from Calbiochem. Knockdown of HIF-1 $\alpha$ in RCC4 was achieved by transfecting cells for $24 \mathrm{~h}$ with small interfering RNA (siRNA) using G-fectin (Genolution Pharmaceuticals, Seoul, Republic of Korea), as described by the manufacturer.

\section{Determination of oxygen consumption rate}

Oxygen consumption rate (OCR) was analyzed using an XF24 analyzer (Seahorse, MA, USA). RCC4 or RCC4/VHL cells were plated at $5 \times 10^{3}$ cells/well and incubated with DMSO or $100 \mu \mathrm{M}$ auraptene for $24 \mathrm{~h}$. After measuring basal OCR, the mitochondrial ATP synthase inhibitor, oligomycin $(2 \mu \mathrm{g} / \mathrm{ml})$, was added, followed sequentially by addition of the mitochondrial uncoupler, carbonyl cyanide 3-chlorophenylhydrazone (CCCP; $5 \mu \mathrm{M}$ ), and the mitochondrial complex I inhibitor, rotenone $(2 \mu \mathrm{M})$. OCR was measured after addition of each agent.

\section{Measurement of cell viability}

RCC4 cells were seeded in 96-well plates and incubated in media containing different concentrations of auraptene $(0,25,50,75$ and $100 \mu \mathrm{M})$ for 6,12 and $24 \mathrm{~h}$ at $37^{\circ} \mathrm{C}$ under normoxic $\left(5 \% \mathrm{CO}_{2} / 21 \% \mathrm{O}_{2}\right)$ conditions. The media were removed and serum-free medium

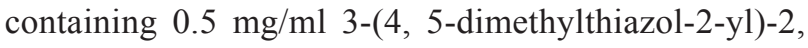


5-diphenyltetrazolium bromide (MTT) was added. After $1 \mathrm{~h}$, the MTT-containing media were removed and DMSO was added to dissolve formazan crystals. Absorbance was measured at $570 \mathrm{~nm}$ using a Multiskan Ascent plate reader (Thermo Scientific, MA, USA). In the sulforhodamine B assay, RCC4 cells $\left(4 \times 10^{3}\right.$ cells per well) were seeded in triplicate in 96-well plates and incubated overnight. To each well as added medium containing $0,25,50,75$ and $100 \mu \mathrm{M}$ auraptene, followed by incubation for 12 or $24 \mathrm{~h}$. The media were discarded and cells were fixed with $10 \%$ TSA at $4^{\circ} \mathrm{C}$ for $1 \mathrm{~h}$. After washing with distilled water, the cells were incubated with $0.4 \%$ SRB (Sigma-Aldrich, SG, Switzerland) solution at room temperature for $20 \mathrm{~min}$. The plates were washed with $1 \%$ acetic acid five times and dried in air. Proteins were resolved with $10 \mathrm{mM}$ unbuffered Tris and absorbance was read at $490 \mathrm{~nm}$ using a Multiskan Ascent plate reader.

\section{RNA isolation and real-time PCR}

Total RNA was isolated from cells using isolRNA lysis reagent (5 PRIME, CA, USA). cDNA way synthesized from total RNA by reverse transcription using an M-MLV reverse transcriptase system (Invitrogen, CA, USA), according to the manufacturer's instructions. Realtime polymerase chain reaction (PCR) was performed using a SYBR mix and a Rotor-Gene 6000 RealTime Rotary Analyzer system (Corbett Life Science, Venlo, Netherlands). The following primer pairs were used: HIF-1 $\alpha, 5^{\prime}$-CCA CCT ATG ACC TGC TTG GT-3' (forward) and 5'-TAT CCA GGC TGT GTC GAC TG-3' (reverse); LDHA, 5'-TGT GCC TGT ATG GAG TGG AA3' (forward) and 5'-AGC ACT CTC AAC CAC CTG CT3' (reverse); GLUT1, 5'-GCC CTG GAT GTC CTA TCT GA-3' (forward) and 5'-CCC ACG ATG AAG TTT GAG GT-3' (reverse); HK2, 5'-TAG GGC TTG AGA GCA CCT GT-3' (forward) and 5'-CCA CAC CCA CTG TCA CTT TG-3' (reverse); VEGF, 5'-CCT TGC TGC TCT ACC TCC AC-3' (forward) and 5'-CAC ACA GGA TGG CTT GAA GA-3' (reverse); and $\beta$-actin, 5'-GTC GTA CCA CTG GCA TTG TG-3' (forward) and 5'-CTC TCA GCT GTG GTG GTG AA-3' (reverse); $P F K, 5^{\prime}$-GAA GAG CCC TTC GAC ATC AG-3' (forward) and 5' - TCT TCC TGC AGT CAA ACA CG -3' (forward).

\section{Wound-healing assay}

RCC4 cells were cultured in the presence or absence of auraptene, then, monolayers were scratched with a pipet tip. Gap width was measured after $24 \mathrm{~h}$ using ImageJ program.

\section{Endothelial cell tube-formation assay}

Human umbilical vein endothelial cells (HUVECs) in EBM-2 media supplemented with $0.1 \%$ FBS were plated on Matrigel basement membranes (BD Biosciences, NJ, USA) and incubated for $8 \mathrm{~h}$ under hypoxic conditions. Auraptene was added to media, after which cells were stained with calcein-AM (SigmaAldrich, SG, Switzerland). Intracellular calcein-AM fluorescence ( $\mathrm{n}=10$ slides/condition) was imaged using an IX70 fluorescence microscope (Olympus, Tokyo, Japan).

\section{Matrigel plug assay}

Matrigel (BD Bioscience, MA, USA) was thawed on $4^{\circ} \mathrm{C}$, then, $400 \mu \mathrm{l}$ of Matrigel was mixed with $200 \mu \mathrm{l}$ serum-free DMEM media, 50 ng VEGF (R\&D Systems, MN, USA) and $40 \mathrm{u}$ heparin (Huons, Seongnam, Republic of Korea). DMSO or $100 \mathrm{uM}$ auraptene was added to the mixture. Then, Matrigel mixture was subcutaneously injected to the flank of 6 weeks old male immunedeficient mice ( $n=5$, each group). Mouse were purchased from Japan SLC (SLC, Hamamatsu, Japan) and kept in pathogen free facility. Animal experiment was approved by the Institutional Animal Care and Use Committee of Chungnam National University. The ethical approval number is CNU-00356. Neovascularization was quantified by measuring hemoglobin content using Drabkin's reagents (Sigma-Aldrich, SG, Switzerland) after 7 days of injection. After removing plug from skin, plug was homogenized at $4^{\circ} \mathrm{C}$ for 18 hours and centrifuged at $10,000 \mathrm{rpm}$ at $4^{\circ} \mathrm{C} .5 \mathrm{ml}$ of Drabkin's solution was added to the supernatant and incubated at RT for 15 minutes. The absorbance at $540 \mathrm{~nm}$ was measured in ELISA plate reader (Molecular Devices, CA, USA).

\section{Xenograft model}

To generate RCC4 xenografts, $1 \times 10^{7}$ cells in $100 \mu \mathrm{M}$ serum free DMEM were mixed with an equal volume of Matrigel (BD Biosciences, NJ, USA), and the mixture was injected subcutaneously into 5 week old male nude mice ( $n=10$ per group). Beginning 9 days later, auraptene or DMSO diluted in $50 \mu$ saline was intratumorally injected every other day. Tumor size was measured with calipers and tumor volume was calculated using the formula: width ${ }^{2} x$ length/2. The mice were sacrificed and the tumor tissue was fixed in 4\% paraformaldehyde for immunohistochemistry.

\section{Immunohistochemistry}

Tumors of DMSO- or auraptene- treated mice was fixed with 4\% paraformaldehyde and paraffin embedded. Sections were prepared for hematoxylin and eosin (H\&E) staining. After deparaffinization, sections were stained with Harris hematoxylin for 4 minutes. Then, sections were washed with running water 4 min, stained with eosin for $2 \mathrm{~min}$ and serial washing with ethanol and xylene was proceeded. For CD31 immunofluorescence staining, 
sections were deparaffinized and blocked for $30 \mathrm{~min}$ with protein blocking buffer (DAKO, Glostrup, Denmark). After incubation with CD31 antibody (Abcam, MA, USA), sections were washed with TBST buffer and incubated with secondary fluorescence antibody. Intracellular CD31 fluorescence ( $n=10$ slides/ condition) was visualized using an IX70 fluorescence microscope (Olympus, Tokyo, Japan).

\section{Western blotting}

Cells were lysed with RIPA buffer (50 mM Tris$\mathrm{HCl} \mathrm{pH} 7.5,150 \mathrm{mM} \mathrm{NaCl}, 1 \%$ Nonidet P-40, 0.5\% deoxycholate, $0.1 \%$ SDS) containing protease inhibitor cocktail (Roche, Basel, Switzerland), with or without phosphatase inhibitor (Roche). Equal amounts of protein were resolved by sodium dodecyl sulfate-polyacrylamide gel electrophoresis (SDS-PAGE) and then transferred to nitrocellulose membranes (Pall Corporation, NY, USA). Membranes were probed first with anti-HIF-1 $\alpha$ (Novus Biologicals, CO, USA), anti-rabbit $\beta$-actin (Santa Cruz Biotechnology, CA, USA), anti- $\alpha$-tubulin (Sigma-Aldrich, SG, Switzerland), anti-rabbit eIF $2 \alpha$ (MyBioSource, CA, USA), and anti-rabbit phosphoeIF2 $\alpha$ (Merck Millipore, MA, USA) primary antibodies, and then with horseradish peroxidase (HRP)-conjugated anti-mouse IgG (Pierce Biotechnology, MA, USA) or anti-rabbit IgG (Calbiochem, MA, USA) secondary antibody, as appropriate. Immunoreactive proteins were detected using an enhanced chemiluminescence (ECL) system (Amersham Biosciences, Buckinghamshire, England).

\section{Statistical analysis}

Results are presented as means \pm standard deviation (SD) of triplicate determinations. The statistical significance of differences between two means was determined by one-tailed Student's $t$-test. A $P$-value $<0.05$ was considered significant; $P$-values for specific comparisons are indicated in figure legends.

\section{ACKNOWLEDGMENTS AND FUNDING}

This work was supported by the National Research Foundation of Republic of Korea (NRF) grant funded by the Ministry of Science, ICT \& Future Planning (MSIP) (2012R1A2A2A01046957, 2014R1A2A1A11051231, 2014R1A1A1037655) and research fund of Chungnam National University.

\section{CONFLICTS OF INTEREST}

The authors have declared that no conflict of interests exist.

\section{REFERENCES}

1. Benhaim R, Oussoultzoglou E, Saeedi Y, Mouracade P, Bachellier P, Lang H. Pancreatic metastasis from clear cell renal cell carcinoma: outcome of an aggressive approach. Urology. 2015; 85:135-140.

2. Hamada S, Ito $\mathrm{K}$, Kuroda $\mathrm{K}$, Sato A, Asakuma J, Horiguchi A, Seguchi K, Asano T. Clinical characteristics and prognosis of patients with renal cell carcinoma and liver metastasis. Mol Clin Oncol. 2015; 3:63-68.

3. Erdem S, Yegen G, Telci D, Yildiz I, Tefik T, Issever H, Kilicaslan I, Sanli O. The increased transglutaminase 2 expression levels during initial tumorigenesis predict increased risk of metastasis and decreased disease-free and cancer-specific survivals in renal cell carcinoma. World J Urol. 2014.

4. Sotgia F, Whitaker-Menezes D, Martinez-Outschoorn UE, Flomenberg N, Birbe RC, Witkiewicz AK, Howell A, Philp NJ, Pestell RG, Lisanti MP. Mitochondrial metabolism in cancer metastasis: visualizing tumor cell mitochondria and the "reverse Warburg effect" in positive lymph node tissue. Cell Cycle. 2012; 11:1445-1454.

5. Lu J, Tan M, Cai Q. The Warburg effect in tumor progression: Mitochondrial oxidative metabolism as an antimetastasis mechanism. Cancer Lett. 2015; 356:156-164.

6. Kamarajugadda S, Stemboroski L, Cai Q, Simpson NE, Nayak S, Tan M, Lu J. Glucose oxidation modulates anoikis and tumor metastasis. Mol Cell Biol. 2012; 32:1893-1907.

7. LeBleu VS, O'Connell JT, Gonzalez Herrera KN, Wikman H, Pantel K, Haigis MC, de Carvalho FM, Damascena A, Domingos Chinen LT, Rocha RM, Asara JM, Kalluri R. PGC-1alpha mediates mitochondrial biogenesis and oxidative phosphorylation in cancer cells to promote metastasis. Nat Cell Biol. 2014; 16:992-1003. 1001-1015.

8. Palorini R, Simonetto T, Cirulli C, Chiaradonna F. Mitochondrial complex I inhibitors and forced oxidative phosphorylation synergize in inducing cancer cell death. Int J Cell Biol. 2013; 2013:243876.

9. Chen V, Staub RE, Fong S, Tagliaferri M, Cohen I, Shtivelman E. Bezielle selectively targets mitochondria of cancer cells to inhibit glycolysis and OXPHOS. PLoS One. 2012; 7:e30300.

10. Momose I, Ohba S, Tatsuda D, Kawada M, Masuda T, Tsujiuchi G, Yamori T, Esumi H, Ikeda D. Mitochondrial inhibitors show preferential cytotoxicity to human pancreatic cancer PANC-1 cells under glucose-deprived conditions. Biochem Biophys Res Commun. 2010; 392:460-466.

11. Li J, Mahdi F, Du L, Jekabsons MB, Zhou YD, Nagle DG. Semisynthetic studies identify mitochondria poisons from botanical dietary supplements-geranyloxycoumarins from Aegle marmelos. Bioorg Med Chem. 2013; 21:1795-1803. 
12. Tanaka T, Kohno H, Murakami M, Kagami S, El-Bayoumy K. Suppressing effects of dietary supplementation of the organoselenium 1,4-phenylenebis(methylene) selenocyanate and the Citrus antioxidant auraptene on lung metastasis of melanoma cells in mice. Cancer Res. 2000; 60:3713-3716.

13. Krishnan P, Yan KJ, Windler D, Tubbs J, Grand R, Li BD, Aldaz CM, McLarty J, Kleiner-Hancock HE. Citrus auraptene suppresses cyclin D1 and significantly delays N-methyl nitrosourea induced mammary carcinogenesis in female Sprague-Dawley rats. BMC Cancer. 2009; 9:259.

14. Tanaka T, Kawabata K, Kakumoto M, Matsunaga K, Mori H, Murakami A, Kuki W, Takahashi Y, Yonei H, Satoh K, Hara A, Maeda M, Ota T, Odashima S, Koshimizu K, Ohigashi H. Chemoprevention of 4-nitroquinoline 1-oxide-induced oral carcinogenesis by citrus auraptene in rats. Carcinogenesis. 1998; 19:425-431.

15. Jun DY, Kim JS, Park HS, Han CR, Fang Z, Woo MH, Rhee IK, Kim YH. Apoptogenic activity of auraptene of Zanthoxylum schinifolium toward human acute leukemia Jurkat $\mathrm{T}$ cells is associated with ER stress-mediated caspase- 8 activation that stimulates mitochondria-dependent or -independent caspase cascade. Carcinogenesis. 2007; 28:1303-1313.

16. Jonasch E, Futreal PA, Davis IJ, Bailey ST, Kim WY, Brugarolas J, Giaccia AJ, Kurban G, Pause A, Frydman J, Zurita AJ, Rini BI, Sharma P, Atkins MB, Walker CL, Rathmell WK. State of the science: an update on renal cell carcinoma. Mol Cancer Res. 2012; 10:859-880.

17. Baldewijns MM, van Vlodrop IJ, Vermeulen PB, Soetekouw PM, van Engeland M, de Bruine AP. VHL and HIF signalling in renal cell carcinogenesis. J Pathol. 2010; 221:125-138.

18. Bausch B, Jilg C, Glasker S, Vortmeyer A, Lutzen N, Anton A, Eng C, Neumann HP. Renal cancer in von HippelLindau disease and related syndromes. Nat Rev Nephrol. 2013; 9:529-538.

19. Denko NC. Hypoxia, HIF1 and glucose metabolism in the solid tumour. Nat Rev Cancer. 2008; 8:705-713.

20. Bardos JI, Ashcroft M. Hypoxia-inducible factor-1 and oncogenic signalling. Bioessays. 2004; 26:262-269.

21. Stubbs M, Griffiths JR. The altered metabolism of tumors: HIF-1 and its role in the Warburg effect. Adv Enzyme Regul. 2010; 50:44-55.

22. Cui SY, Huang JY, Chen YT, Song HZ, Huang GC, De W, Wang R, Chen LB. The role of Aurora A in hypoxiainducible factor 1alpha-promoting malignant phenotypes of hepatocelluar carcinoma. Cell Cycle. 2013; 12:2849-2866.

23. Jang M, Kim SS, Lee J. Cancer cell metabolism: implications for therapeutic targets. Exp Mol Med. 2013; 45:e45.

24. Carew JS, Esquivel JA 2nd, Espitia CM, Schultes CM, Mulbaier M, Lewis JD, Janssen B, Giles FJ, Nawrocki ST. ELR510444 inhibits tumor growth and angiogenesis by abrogating HIF activity and disrupting microtubules in renal cell carcinoma. PLoS One. 2012; 7:e31120.

25. Wang Z, Dabrosin C, Yin X, Fuster MM, Arreola A, Rathmell WK, Generali D, Nagaraju GP, El-Rayes B, Ribatti D, Chen YC, Honoki K, Fujii H, Georgakilas AG, Nowsheen S, Amedei A, et al. Broad targeting of angiogenesis for cancer prevention and therapy. Semin Cancer Biol. 2015.

26. Powis G, Kirkpatrick L. Hypoxia inducible factor-1alpha as a cancer drug target. Mol Cancer Ther. 2004; 3:647-654.

27. Semenza GL. HIF-1 mediates metabolic responses to intratumoral hypoxia and oncogenic mutations. J Clin Invest. $2013 ; 123: 3664-3671$.

28. Moore LE, Nickerson ML, Brennan P, Toro JR, Jaeger E, Rinsky J, Han SS, Zaridze D, Matveev V, Janout V, Kollarova H, Bencko V, Navratilova M, SzeszeniaDabrowska N, Mates D, Schmidt LS, et al. Von HippelLindau (VHL) inactivation in sporadic clear cell renal cancer: associations with germline VHL polymorphisms and etiologic risk factors. PLoS Genet. 2011; 7:e1002312.

29. Liu YV, Semenza GL. RACK1 vs. HSP90: competition for HIF-1 alpha degradation vs. stabilization. Cell Cycle. 2007; 6:656-659.

30. Minet E, Mottet D, Michel G, Roland I, Raes M, Remacle J, Michiels C. Hypoxia-induced activation of HIF-1: role of HIF-1alpha-Hsp90 interaction. FEBS Lett. 1999; 460:251-256.

31. Zhu K, Chan W, Heymach J, Wilkinson M, McConkey DJ. Control of HIF-1alpha expression by eIF2 alpha phosphorylation-mediated translational repression. Cancer Res. 2009; 69:1836-1843.

32. Twardowski P, Figlin RA. Bevacizumab plus interferon alpha in patients with metastatic renal cell carcinoma. Nat Clin Pract Oncol. 2008; 5:436-437.

33. Roulin D, Waselle L, Dormond-Meuwly A, Dufour M, Demartines N, Dormond O. Targeting renal cell carcinoma with NVP-BEZ235, a dual PI3K/mTOR inhibitor, in combination with sorafenib. Mol Cancer. 2011; 10:90.

34. Garcia JA, Hutson TE, Elson P, Cowey CL, Gilligan T, Nemec C, Dreicer R, Bukowski RM, Rini BI. Sorafenib in patients with metastatic renal cell carcinoma refractory to either sunitinib or bevacizumab. Cancer. 2010; 116:5383-5390.

35. Hutson TE, Bukowski RM, Cowey CL, Figlin R, Escudier B, Sternberg CN. Sequential use of targeted agents in the treatment of renal cell carcinoma. Crit Rev Oncol Hematol. 2011; 77:48-62.

36. Zhao L, Zhao X, Zhao K, Wei P, Fang Y, Zhang F, Zhang B, Ogata K, Mori A, Wei T. The alpha-tocopherol derivative ESeroS-GS induces cell death and inhibits cell motility of breast cancer cells through the regulation of energy metabolism. Eur J Pharmacol. 2014; 745: 98-107. 
37. McLachlan A, Kekre N, McNulty J, Pandey S. Pancratistatin: a natural anti-cancer compound that targets mitochondria specifically in cancer cells to induce apoptosis. Apoptosis. 2005; 10:619-630.

38. Okuyama S, Yamamoto K, Mori H, Toyoda N, Yoshimura M, Amakura Y, Yoshida T, Sugawara K, Sudo M, Nakajima M, Furukawa Y. Auraptene in the Peels of Citrus kawachiensis (Kawachi Bankan) Ameliorates Lipopolysaccharide-Induced Inflammation in the Mouse Brain. Evid Based Complement Alternat Med. 2014; 2014:408503.

39. Wang L, Williamson SR, Wang M, Davidson DD, Zhang S, Baldridge LA, Du X, Cheng L. Molecular subtyping of metastatic renal cell carcinoma: implications for targeted therapy. Mol Cancer. 2014; 13:39.
40. Koh MY, Spivak-Kroizman T, Venturini S, Welsh S, Williams RR, Kirkpatrick DL, Powis G. Molecular mechanisms for the activity of PX-48, an antitumor inhibitor of the hypoxia-inducible factor-1alpha. Mol Cancer Ther. 2008; 7:90-100.

41. Rapisarda A, Uranchimeg B, Sordet O, Pommier Y, Shoemaker RH, Melillo G. Topoisomerase I-mediated inhibition of hypoxia-inducible factor 1 : mechanism and therapeutic implications. Cancer Res. 2004; 64:1475-1482.

42. Wu B, Teng H, Yang G, Wu L, Wang R. Hydrogen sulfide inhibits the translational expression of hypoxia-inducible factor-1alpha. Br J Pharmacol. 2012; 167:1492-1505.

43. Cowey CL, Rathmell WK. Using Molecular Biology to Develop Drugs for Renal Cell Carcinoma. Expert Opin Drug Discov. 2008; 3:311-327. 